\section{A fast and efficient algorithm for the Fisher exact test}

\author{
JERROLD H. ZAR \\ Northern Illinois University, DeKalb, Illinois
}

The Probability of Observed Frequencies in a $2 \times 2$ Table. The Fisher exact test, as a procedure for obtaining exact probabilities associated with statistical hypotheses about $2 \times 2$ contingency tables, is especially recommended when dealing with small frequencies (e.g., Zar, 1984, pp. 390-395). Let us represent a $2 \times 2$ contingency table like this:

\begin{tabular}{|cc|c}
\hline$f_{11}$ & $f_{12}$ & $R_{1}$ \\
$f_{21}$ & $f_{22}$ & $R_{2}$ \\
\hline$C_{1}$ & $C_{2}$ & $n$
\end{tabular}

where $f_{i j}$ is the observed frequency in row $i$ and column $j$ of the table, $R_{i}$ is the sum of the two frequencies in row $i$, and $C_{j}$ is the sum of the two frequencies in column $j$. Given the observed marginal totals $\left(R_{1}, R_{2}, C_{1}\right.$, and $\left.C_{2}\right)$, the probability of the observed $f_{i j}$ s occurring by chance, under the null hypothesis that row effects are independent of column effects, is

$$
P=\frac{R_{1} ! R_{2} ! C_{1} ! C_{2} !}{f_{11} ! f_{12} ! f_{21} ! f_{22} ! n !} .
$$

Fast and Efficient Computation. The probability associated with a one-tailed test is the probability of the observed table (as obtained by the equation above) added to the sum of the probabilities of all possible tables having the same marginal totals but with frequencies more extreme than in the observed table and in the direction indicated in the alternate hypothesis. Inasmuch as use of this equation requires that nine factorials be calculated for each of several possible tables, computational shortcuts have been proposed in order to reduce the computational time and to avoid computer overflows resulting from attempts to calculate the factorials for moderate to large numbers. I describe here a computer algorithm that employs a combination of particularly useful shortcuts, thus enabling the one-tailed probability to be obtained by a simplified equivalent of the above equation, which only needs to be resorted to once.

Leslie (1955), Sakoda and Cohen (1957), and Ghent (1972) have pointed out that the above equation may be rewritten, equivalently, as

The suggestions of a referee are greatly appreciated. Address correspondence to the author at: Department of Biological Sciences, Northern Illinois University, DeKalb, IL 60115-2861.

$$
P=\frac{\frac{R_{1} !}{f_{11} ! f_{12} ! f_{21} ! f_{22} !}}{\frac{n !}{C_{1} ! C_{2} !}}
$$

which has the product of two binomial coefficients in the numerator and a binomial coefficient in the denominator. It is efficient, therefore, to have a computer subroutine for calculating a binomial coefficient.

The computation of a factorial (call it $y$ !) is very timeconsuming, and for $y>33$ the result is $>10^{38}$, a number larger than can typically be accommodated by microcomputers. We can avoid the necessity of computing three factorials for each binomial coefficient (i.e., nine for the Fisher exact test probability) by calculating the binomial coefficient

$$
\frac{N !}{X !(N-X) !}
$$

as

$$
\frac{(N)(N-1)(N-2) \cdots(X+1)}{(N-X) !}
$$

a calculation made even more efficient by having $N-X<X$. If either the numerator or denominator is in danger of being too large to be stored in the computer, then the latter computation may employ logarithms:

$\ln N+\ln (N-1)+\ln (N-2)+\cdots-\ln [(N-X) !]$,

although this considerably slows down the calculation. Computation may be made even simpler-and much faster-by using the Stirling approximation:

$$
\begin{aligned}
\ln y ! & =\frac{\ln (2 \pi)}{2}+(\ln y)(y+0.5)-y+\frac{1}{12 y} \\
& -\frac{1}{360 y^{3}}+\frac{1}{1260 y^{5}}-\frac{1}{1680 y^{7}}
\end{aligned}
$$

(Davis, 1964 , p. 255 , Eq. 6.16; p. 257, Eq. 6.1.41) to obtain the logarithm of each of the nine factorials referred to above. This approximation is very fast and accurate; only the first four of the seven terms shown are required for accuracy to seven significant figures for $y$ as small as 9. Therefore, factorials can be calculated directly (and rather quickly) for integers up to 8 or 9 , and they can be obtained from the approximation for integers larger than that.

An additional time-saving procedure is that of Feldman and Kluger (1963), who have shown that when one has determined the probability for the table of observed frequencies, the probability of each more extreme table can be obtained by a simple procedure requiring no factorials at all. If we designate the smallest of the four table frequencies as $f_{a}$, and the other three frequencies as $f_{b}, f_{d}$, and $f_{c}$ in clockwise order from $f_{a}$, then the next more ex- 
treme extreme table will have $f_{a}$ and $f_{d}$ each decreased by 1 and $f_{b}$ and $f_{c}$ each increased by 1 . If we refer to these four new frequencies as $f_{a}^{\prime}, f_{b}^{\prime}, f_{d}^{\prime}, f_{c}^{\prime}$, respectively, then the probability of this new table is

$$
P^{\prime}=\frac{f_{a} f_{d}}{f_{b}^{\prime} f_{c}^{\prime}} P \text {. }
$$

Then we use the symbols $f_{a}, f_{b}, f_{c}, f_{d}$, and $P$ in place of $f_{a}^{\prime}, f_{b}^{\prime}, f_{c}^{\prime}, f_{d}^{\prime}$, and $P^{\prime}$, respectively; new values are obtained for $f_{a}^{\prime}=f_{a}-1, f_{b}^{\prime}=f_{b}+1, f_{c}^{\prime}=f_{c}+1$, and $f_{d}^{\prime}=f_{d}-1$; and the above equation is applied again. New values of $f_{a}^{\prime}, f_{b}^{\prime}, f_{c}^{\prime}$, and $f_{d}^{\prime}$ are again determined, and the procedure is repeated as long as the four table frequencies fit within the marginal totals of the original table. Then all the probabilities computed in this fashion are summed, arriving thereby at the one-tailed probability for the test. This probability (call it $P_{1}$ ) is the smaller of the region of the probability distribution extending from the probability of the original table (call it $P_{0}$ ) to the extreme of one tail and the region extending from the original table's probability to the extreme of the other tail. If the hypothesis being tested calls for the other possible onetailed probability, the latter may be obtained as

$$
1-P_{1}-P_{0} \text {. }
$$

The two-tailed Fisher exact test addresses the same statistical hypotheses as does $2 \times 2$ contingency table testing by chi-square or the log-likelihood ratio (e.g., Zar, 1984, pp. 64-66, 71-72). If either the two row totals are the same or the two column totals are the same, then the two-tailed probability is two times the one-tailed probability (Ghent, 1972). If, however, $R_{1} \neq R_{2}$ and/or $C_{1} \neq C_{2}$, then the probability $P_{1}$ above is to be added to the appropriate one-tailed probability from the other tail, which is determined as follows. Examine the most extreme (i.e., the last) table computed in first tail: call the smallest marginal total $m_{1}$ and call the smaller of the two totals in the opposite margin $m_{2}$. Then let us call the frequency associated with marginal frequencies $m_{1}$ and $m_{2}$ frequency $f$; change it to $m_{1}-f$, and define the other three frequencies as those adding up to the four marginal totals. The table thus formed is the most extreme table in the second tail, and its probability is computed by the binomial coefficient shortcut. If this probability is greater than the probability of the originally observed table, then it is ignored and the probability of the two-tailed test is declared the same as that for the one-tailed test; if it is not, then we form the immediately less extreme table in the second tail, as follows. If we refer to the smallest frequency in the table last formed as $f_{a}^{\prime}$, and the other three frequencies as $f_{b}^{\prime}, f_{d}^{\prime}$, and $f_{c}^{\prime}$ (clockwise from $f_{a}^{\prime}$ ), then the next table to examine is that in which $f_{a}^{\prime}$ and $f_{d}^{\prime}$ are each increased by 1 and $f_{b}^{\prime}$ and $f_{c}^{\prime}$ are each decreased by 1. (Let us refer to these new values as $f_{a}, f_{b}, f_{d}$, and $f_{c}$.) The probability of this newly formed table may be obtained as

$$
\boldsymbol{P}=\frac{f_{b}^{\prime} f_{c}^{\prime}}{f_{\mathrm{a}} f_{d}} \boldsymbol{P}^{\prime},
$$

where $\boldsymbol{P}^{\prime}$ is the probability of the preceding table in the second tail (i.e., the probability that is next most extreme in that tail). This procedure of forming a new table and applying the above equation is continued as long as the resultant $P$ is $\leq$ the probability of the original table. Then, all the $P$ s calculated in the second tail are summed and added to $P_{1}$, the probability described above for the onetailed test.

Program Availability. A listing of a portable BASIC program employing the above procedures is available from the author, at no charge. The program computes the probability appropriate for either a one-tailed or a twotailed test, as specified by the user. The input consists of the four observed frequencies and an indication of whether a one-tailed or two-tailed test probability is desired. The output gives the row, column, and grand totals, the four cell frequencies and probability of each table examined, and any of the other probabilities discussed above that are appropriate.

\section{REFERENCES}

Davis, P. J. (1964). Gamma function and related functions. In M. Abramowitz \& I. A. Stegun (Eds.), Handbook of mathematical functions (pp. 253-293). Washington, DC: National Bureau of Standards.

Feldman, S. E., \& Kluger, E. (1963). Short cut calculation of the Fisher-Yates "exact test." Psychometrika, 2, 289-291.

Ghent, A. W. (1972). A method for exact testing of $2 \times 2,2 \times 3,3 \times 3$, and other contingency tables, employing binomial coefficients. American Midland Naturalist, 88, 15-27.

LeSLIE, P. H. (1955). A simple method of calculating the exact probability in $2 \times 2$ contingency tables with small marginal totals. Biometrika, 42, 522-523.

SAKodA, J. M., CoHEN, B. H. (1957). Exact probabilities for contingency tables using binomial coefficients. Psychometrika, 22, 83-86.

ZaR, J. H. (1984). Biostatistical analysis (2nd ed.). Englewood Cliffs, NJ: Prentice-Hall.

(Revision accepted for publication February 9, 1987.) 